\title{
Validación del Cuestionario de Apoyo Social Comunitario en Personas Mayores Chilenas
}

\section{Validation of the Community Support Questionnaire among Elderly Chilean People}

\author{
Lorena P. Gallardo-Peralta ${ }^{1}$ y José Luis Gálvez-Nieto ${ }^{2}$
}

\begin{abstract}
Resumen
La comunidad es un recurso psicosocial que promueve el bienestar en la vejez. En este artículo se presenta la validación del cuestionario de apoyo social comunitario en una muestra representativa de personas mayores chilenas $(\mathrm{n}=777)$. Los resultados de esta investigación confirman el modelo original de tres factores (Gracia, Herrero, \& Musitu, 2002), a saber: integración comunitaria, participación comunitaria y apoyo social de los sistemas formales. Los tres factores explicaron en conjunto el $82 \%$ de la varianza del constructo. Este cuestionario constituye una herramienta para la intervención social gerontológica de cara a la prevención del aislamiento o falta de integración social de los mayores.
\end{abstract}

Palabras clave: apoyo social comunitario, personas mayores, cuestionario, propiedades psicométricas

\begin{abstract}
The community is a psychosocial resource that promotes well-being in old age. This article presents the validation of the Questionnaire of Community Social Support in a representative sample of elderly Chilean people $(n=777)$. The results of this research confirm the original model of three factors (Gracia, Herrero, \& Musitu, 2002), namely: community integration, community participation and social support from formal systems. The three factors explained together $82 \%$ of the variance of the construct. This questionnaire constitutes a tool for gerontological social intervention in order to prevent the isolation or lack of social integration of the elderly.
\end{abstract}

Keywords: community social support, elderly people, questionnaire, psychometric properties

Agradecimientos: Esta investigación ha sido financiada por el Fondo Nacional de Desarrollo Científico y Tecnológico (FONDECYT N ${ }^{\circ} 11140020$ ) del Gobierno de Chile y Universidad de Tarapacá (Proyecto UTA Mayor 3762-17).

\footnotetext{
${ }^{1}$ Doctora en Trabajo Social. Académica de la Escuela de Trabajo Social de la Universidad de Tarapacá, Arica, Chile. Investigadora visitante en el Instituto de Economía, Geografía y Demografía del Consejo Superior de Investigaciones Científicas (CSIC), Madrid, España. Correo: lgallardo@uta.cl

${ }^{2}$ Doctor en Ciencias de la Educación. Académico del Departamento de Trabajo Social de la Universidad de La Frontera, Temuco, Chile. Correspondencia: Departamento de Trabajo Social de la Universidad de La Frontera. Avenida Francisco Salazar 01145, Temuco, Chile. Correo: jose.galvez@ufrontera.cl
} 


\section{Introducción}

Chile experimenta un acelerado proceso de envejecimiento de su población, lo que es sin duda supone un avance social y sanitario. La última encuesta CASEN (2015) sitúa el envejecimiento de la población chilena en un 17.5\%. Ello explica el aumento sustantivo de la actividad científica nacional en torno al envejecimiento y la vejez. Específicamente, el interés se orienta a conocer los elementos que promueven el bienestar en la vejez (Bonout et al., 2012; Castillo-Carniglia, Albala, Gangour, \& Uauy, 2012; Gallardo-Peralta, 2017; Herrera, Barros, \& Fernández, 2011; Mella et al., 2004; Torres, Quezada, Rioseco, \& Ducci, 2008; Urzúa, Bravo, Ogalde, \& Vargas, 2011; Vivaldi \& Barra, 2012). Al hilo de esta premisa, la integración social de las personas mayores es un ámbito ampliamente investigado por su potencial en el bienestar de este grupo de edad (Antonucci, Ajrouch, \& Birditt, 2014) o dicho de otro modo el bienestar se relaciona positivamente con disponer de mejores relaciones sociales (DomínguezGuedea, 2016). Sin embargo, el análisis suele centrarse en la integración en redes familiares $u$ otro tipo de vínculos íntimos, y no se profundizan la integración social en la comunidad (Gracia \& Herrero, 2006; Heinze, Kruger, Reischl, Cupal, \& Zimmerman, 2015). De esta manera, este artículo tiene como objetivo validar un cuestionario de apoyo social comunitario en una muestra representativa de personas mayores chilenas que residen en el extremo norte de Chile. Entendiendo que la comunidad, como fuente de apoyo social, tiene un rol trascendental en el bienestar de las personas mayores chilenas, de allí, la relevancia de contar con instrumentos internacionalmente reconocidos en la evaluación de la integración social comunitaria.

\section{Redes sociales en la vejez}

Se ha discutido ampliamente en el ámbito de las ciencias sociales y del comportamiento los cambios que experimentan las redes de apoyo social en la vejez (Antonucci, Ajrouch, \& Birditt, 2014; Chappell \& Funk, 2011; Fiori, Smith \& Antonucci, 2007). En general los resultados muestran que las redes sociales tienden a disminuir a medida que las personas envejecen, sin embargo, los denominados contactos sociales significativos permanecen estables (Lang \& Carstensen, 1994). Tal como afirman Carstensen, Isaacowitz y Charles (1999) la reducción de estos contactos sociales en la vejez es un proceso altamente selectivo, las personas mayores tienden a sacrificar los contactos superficiales e intentan mantener aquellos más cercanos, es decir, aquellos nodos sociales que le proporcionan mayor recompensa emocional. Así los aspectos estructurales que caracterizan las redes sociales en la vejez, suelen ser: redes sociales de tamaño reducido (Cloutier-Fisher et al. 2011) y de composición primordialmente familiar (GallardoPeralta, 2013). No obstante, el reciente estudio de Nguyen (2017) identifica cuatro tipologías de redes sociales en la vejez: diversa, centrada en la familia, no focalizada y restringida. Quienes disponen de redes restringidas tienen más probabilidades de experimentar sentimientos de soledad y aislamiento (Burholt \& Dobbs, 2014). Por el contrario, las redes sociales diversas ofrecen una mayor integración social a la persona mayor (Nguyen, 2017). Por ello se plantea que la heterogeneidad en la composición de la red le permite a la persona mayor acceder a diversos tipos de apoyo social, que pueden complementarse, en situaciones de crisis o bien otorgar mayor bienestar (Cheng, Lee, Chan, Leung, \& Lee, 2009; Fiori, Antonucci, \& Akiyama, 2008; Li \& Zhang, 2015; Litwin \& Shiovitz-Ezra, 2011).

\section{La Comunidad como red de apoyo social}

Las redes comunitarias pueden por tanto ser una alternativa complementaria al apoyo social de las redes familiares o íntimas (redes primarias), pero también puede ser en sí misma la red más significativa para la persona mayor. Al respecto, en Chile la participación comunitaria (en diversos tipos de agrupaciones) en personas mayores de 60 años equivale a un $32 \%$ y los hallazgos muestran una vinculación significativa con el bienestar (Herrera, Elgueta, \& Fernández, 2014).

El potencial de la comunidad como un recurso psicosocial se debe a su carácter de red amplia y heterogénea, al estar compuesta por miembros de agrupaciones, organizaciones o grupos religiosos, deportivos, culturales o de la comunidad (por ejemplo, los vecinos). Además, la integración en 
la comunidad se caracteriza por la participación voluntaria, un menor grado de compromiso, por cuanto el individuo puede entrar y salir de este espacio social y, además, es un espacio de menor intimidad, en términos de que sus miembros pueden o no conocer personalmente al otro (Thoits, 2011). Pero su principal fortaleza es la posibilidad de proporcionar al individuo un sentimiento de pertenencia a una estructura social amplia y un sentido de identidad social (Gracia \& Herrero, 2006).

\section{Sobre la evaluación del apoyo social comunitario}

En Chile no se ha diseñado y tampoco se ha validado un cuestionario que valore específicamente el apoyo social de la comunidad. No obstante, lo que suele ser evaluado es la participación en agrupaciones o asociaciones de carácter comunitario, así, entre las encuestas más representativas de las personas mayores en Chile se incluyen preguntas sobre participación social, tales como: Encuesta Nacional de Caracterización Socioeconómica (CASEN, 2015) y la Encuesta Nacional de Calidad de Vida (2013), de esta manera se pueda acceder a información relacionada con el número de personas mayores en Chile que participan en grupos sociales y al tipo de agrupación social, pero no se obtiene información sobre los aspectos funcionales de estas redes sociales.

Ahora bien, en Chile solo se ha validado la Escala Multidimensional de Apoyo Social de Zimet, Dahlem, Zimet, \& Farley (1988) en personas mayores chilenas (Arechavala \& Miranda, 2002) y en pacientes diabéticos tipo 2 (Ortiz \& Baeza, 2011). Si bien la validación en personas mayores consultó sobre las redes familiares, amigos y otros significativos, su análisis se centra en el apoyo social percibido de las redes íntimas. Aunque pudiera darse que los "otros significativos" pueden ser vecinos, miembros de organizaciones de autoayuda, profesionales o proveedores de servicios, en este estudio esto no se especifica.

Ante este vacío, se ha decidido optar por un instrumento que valore de manera amplia la integración y la participación comunitaria, como es el caso de Cuestionario de Apoyo Social Comunitario (Gracia, Herrero, \& Musitu, 2002).
Como advertíamos, la comunidad es un espacio reciprocidad del apoyo social, por tanto, las personas pueden acceder y también proporcionar diversos tipos de apoyo, ya sea emocional, instrumental o bien informativo. En este sentido, el cuestionario que se validará en esta investigación valora distintos aspectos del apoyo social en la comunidad, tales como: integración en el barrio o comunidad de vecinos, participación en actividades de esta comunidad de vecinos y finalmente, el apoyo social de los sistemas formales de la comunidad. Al respecto, la evidencia empírica confirma que la integración/participación con los vecinos incrementa el bienestar en la vejez (Brown et al. 2009; Hand, Law, Hanna, Elliott, \& McColl, 2012). Asimismo, el apoyo social de los sistemas formales influye positivamente en la calidad de vida de los mayores (van Bilsen, Hamers, Groot, $\&$ Spreeuwenberg, 2008).

\section{Estructura teórica y factorial del cuestionario de apoyo social comunitario}

La medida de apoyo social comunitario es un instrumento que evalúa el grado de integración y participación comunitaria, así como un índice del apoyo percibido en organizaciones formales de la comunidad (Gracia \& Herrero, 2006).

Este instrumento se constituye como una medida útil para la evaluación psicosocial de la comunidad y de ámbitos vinculados a la salud pública. En el ámbito aplicado, resulta una medida útil para la evaluación de intervenciones comunitarias, tanto en el contexto profesional como en la investigación académica.

En términos de estructura factorial, la escala de apoyo social comunitario posee tres factores denominados integración en la comunidad, participación en la comunidad y apoyo social de los sistemas formales comunitarios. Dicha estructura fue puesta a prueba a través de un estudio psicométrico (Gracia \& Herrero, 2006), realizado principalmente a través de análisis factorial exploratorio, que entregó evidencia suficiente para avalar la presencia de tres factores correlacionados, que en total tuvieron un $48.88 \%$ de la varianza total explicada -análisis de componentes principales-. Otro estudio psicométrico (Herrero \& Gracia, 2007), que aportó evidencia psicométrica más robusta mediante un diseño no experimental longitudinal, 
demostró que el instrumento mantenía la estructura de tres factores correlacionados referidos anteriormente, a saber; integración a la comunidad, participación en la comunidad y apoyo social de los sistemas formales de la comunidad.

Resumiendo, se ha demostrado empíricamente el efecto positivo del apoyo social comunitario en el bienestar de las personas mayores. Dada la relevancia social de este tipo de apoyo social, este estudio tiene como objetivo evaluar las propiedades psicométricas del cuestionario de apoyo social comunitario en una muestra representativa de personas mayores chilenas.

\section{Método}

\section{Participantes}

La muestra está conformada por 777 personas mayores chilenas que residen en la región de Arica y Parinacota (XV), en el extremo norte de Chile. Se trata de un muestreo no probabilístico por disponibilidad, sin embargo, este estudio buscó ser representativo de la población mayor de 60 años de la región XV y para asegurar la representatividad, se recurrió a un muestro estratificado por sexo, etnia y lugar de residencia (rural y urbano). Las características fundamentales de la muestra se recogen en la Tabla 1. De dichos datos conviene destacar la edad media (69.93 años; DT=7.12) y la presencia en la muestra de un porcentaje de personas mayores pertenecientes a una etnia originaria $(30 \%)$. Siendo la etnia Aymara la más frecuente (88\% de los casos).

\section{Procedimiento}

Se realizó un estudio piloto con 33 personas mayores y las preguntas fueron comprendidas por las personas mayores indígenas (10\%) y no indígenas, por tanto, no se realizaron cambios en la redacción de los diversos ítems. Posteriormente, entre los meses de junio a agosto de 2015 se dio paso al trabajo de campo. La aplicación del cuestionario se realizó a través de entrevista personal con una duración de 30 a 40 minutos, los cuestionarios fueron aplicados por profesionales de las disciplinas de Trabajo Social y Psicología. Esta investigación es parte del proyecto FONDECYT 11140020, por lo cual el Comité de Ética de la Universidad de Tarapacá supervisó
Tabla 1. Características de los participantes

\begin{tabular}{lcc}
\hline Variable & Categorías & $\mathrm{n}(\%)$ \\
\hline Sexo & Mujer & $488(63 \%)$ \\
& Hombre & $289(37 \%)$ \\
Edad por & $60-69$ años & $430(55 \%)$ \\
grupos & $70-79$ años & $260(34 \%)$ \\
& $80+$ años & $87(11 \%)$ \\
Estado & Casado/a o conviviente & $370(48 \%)$ \\
conyugal & Soltero/a o divorciado/a & $228(29 \%)$ \\
& $\quad$ Viudo/a & $179(23 \%)$ \\
& Urbana (Arica) & $677(87 \%)$ \\
Residencia & Rural: Altiplano & $58(8 \%)$ \\
& Rural: Valles pre \\
& cordilleranos & $42(5 \%)$ \\
& Ed. Básica incompleta & $70(9 \%)$ \\
Educación & Ed. Básica & $319(41 \%)$ \\
& Ed. Media & $319(41 \%)$ \\
Situación & Ed. Superior & $69(9 \%)$ \\
laboral & Sigue trabajando & $350(45 \%)$ \\
Etnicidad & No trabaja & $427(55 \%)$ \\
& Indígenas (aymara) & $232(30 \%)$ \\
& No indígenas & $545(70 \%)$ \\
\hline
\end{tabular}

y aprobó los aspectos éticos del estudio. De especial importancia es señalar que se obtuvo por escrito el consentimiento informado de todas las personas participantes.

\section{Instrumento}

Apoyo Social Comunitario. Se aplicó el Cuestionario de Apoyo Social Comunitario de Gracia, Herrero y Musitu (2002). Este cuestionario tiene un total de 14 ítems distribuidos en tres escalas: (1) Integración a la comunidad. Esta escala de 4 ítems evalúa la integración del sujeto a su barrio o comunidad próxima (vecinos, vecindario) a través de afirmaciones como "me siento identificado con mi barrio". (2) Participación en la comunidad. Esta escala de 5 ítems valora la participación en el barrio o comunidad más próxima mediante afirmaciones como "participo en las actividades sociales de mi barrio o comunidad". (3) Apoyo social en los sistemas formales. Esta escala de 5 ítems proporciona información sobre la percepción del sujeto, en términos de apoyo social, respecto a los recursos formales de la comunidad: centro de salud familiar, hospital, oficinas comunales, ministeriales o distintos servicios públicos. Se evalúa a través de afirmaciones como "estas organizaciones y servicios son una importante fuente de apoyo". Las categorías de respuesta para 
todas las preguntas es de tipo Likert: (1) muy en desacuerdo a (5) muy de acuerdo. Se obtienen puntuaciones para cada escala evaluada. El índice de consistencia interna (alpha de Cronbach) para el inventario general fue de .63 .

\section{Análisis de datos}

Con el objetivo de entregar evidencias de validez y fiabilidad del cuestionario de apoyo comunitario percibido, se utilizó el método de mitades partidas (cross sample) y se analizó la estabilidad de la estructura factorial, se optó por dividir la muestra en dos mitades aleatorias (n1=388 y n2=389). Para asegurar la equivalencia entre ambas muestras, se calcularon medidas de asociación para hombres y mujeres $\left(\chi^{2}\right.$ $[g l=1]=1.192 ; p=.157)$ y diferencias de medias para la variable edad (t-test $[\mathrm{gl}=775]=.443$; $p=.658$ ), en ambos casos se demostró que las variables son independientes de la selección aleatoria de la muestra.

Para entregar evidencias de validez, se seleccionó la primera muestra $(n 1=388)$ y se realizaron análisis factoriales exploratorios (AFE) con el programa FACTOR versión 10.3 (LorenzoSeva \& Ferrando, 2006). El procedimiento seleccionado para determinar el número de factores fue el método de Hull (Lorenzo-Seva, Timmerman, \& Kiers, 2011), por otra parte, considerando la naturaleza ordinal de las variables, la matriz de correlaciones policórica (Freiberg, Stover, de la Iglesia, \& Fernández, 2013). En base a la propuesta teórica y los resultados empíricos del estudio original (Gracia \& Herrero, 2006), se asumió relación entre los factores, optando por una rotación oblicua, método promin (Lorenzo-Seva, 2011). Finalmente, el método de extracción fue Unweighted Least Squares (ULS). Con la segunda muestra $(\mathrm{n} 2=389)$ se realizó un análisis factorial confirmatorio (AFC) con el programa MPLUS (Muthén \& Muthén, 2012). La opción utilizada para el AFC fue la matriz de correlaciones policórica, ideal para el modelamiento de datos categóricos. Para la estimación de los índices de bondad de ajuste, se utilizó el método Weighted Least Square (WLSMV). Este método permite la obtención de índices robustos, así como estimaciones apropiadas de los parámetros y su nivel de error (Flora \& Curran, 2004). El modelo
AFC fue evaluado a partir de los siguientes índices de bondad de ajuste; chi-cuadrado, índice de ajuste comparativo (CFI), índice de TuckerLewis (TLI) y Error cuadrático medio de aproximación (RMSEA). Para los índices CFI y TLI se consideran ajustes razonables del modelo valores mayores o iguales a .90 (Schumacher \& Lomax, 1996), mientras que para RMSEA un valor inferior a .08 (Browne \& Cudeck, 1996). Para la evaluación de fiabilidad del instrumento, se utilizó el Coeficiente Alfa de Cronbach, sin embargo, considerando las limitaciones de este coeficiente (Trizano-Hermosilla \& Alvarado, 2016), se optó por complementar este análisis mediante el coeficiente Omega de McDonald (McDonald, 1999).

\section{Resultados}

\section{Análisis factorial exploratorio}

Teniendo en cuenta los antecedentes empíricos (Gracia \& Herrero, 2006), que planteaban la existencia de tres factores latentes, se procedió a realizar un AFE. Los resultados de la prueba de adecuación muestral KMO (.814) y la prueba de esfericidad de Bartlett $\left(\mathrm{X}^{2}\right.$ $[\mathrm{gl}=105]=4887.4 ; p<.001)$ avalan la factibilidad de realizar un AFE. Los resultados muestran la presencia tres factores correlacionados, que explicaron un $54.3 \%$ de la varianza del constructo de apoyo comunitario percibido. Sin embargo, al realizar un análisis de la matriz de cargas factoriales, el ítem 1 "Me encuentro a disgusto dentro de mi barrio o comunidad", el ítem 4 "Muy pocas personas de mi comunidad saben quién soy yo", el ítem 9 "No participo en reivindicaciones sociales en mi barrio" y el ítem 13 "Estos servicios no me inspiran la suficiente confianza" presentan unas saturaciones menores a .30 , valor considerado insuficiente para ser parte de la solución factorial.

Considerando este resultado, se optó por realizar un nuevo análisis factorial, eliminando los ítems señalados y seleccionando aquellos que presentaron un adecuado ajuste psicométrico. Nuevamente se analizaron los resultados de la prueba KMO (.818) y la prueba de esfericidad de Bartlett $\left(\mathrm{X}^{2}[\mathrm{gl}=45]=2344.5 ; p<.001\right)$. El segundo AFE permitió comprobar la existencia de tres 
Tabla 2. Análisis factorial exploratorio (n1=388)

\begin{tabular}{|c|c|c|c|c|c|}
\hline \multirow[b]{2}{*}{ Ítems } & \multirow[t]{2}{*}{ Media } & \multirow[t]{2}{*}{ Desv. Tip. } & \multicolumn{3}{|c|}{ Saturaciones factoriales exploratorias } \\
\hline & & & Integración & Apoyo Social & Participación \\
\hline 1.- Me siento identificado con mi barrio & 3.61 & 1.290 & .916 & .017 & -.061 \\
\hline 2.- Mis opiniones son bien recibidas en mi barrio & 3.38 & 1.295 & .793 & .027 & .110 \\
\hline 3.- Siento el barrio como mío & 3.51 & 1.321 & .820 & -.021 & .047 \\
\hline $\begin{array}{l}\text { 4.- Colaboro en las organizaciones y asociaciones } \\
\text { de mi comunidad }\end{array}$ & 2.76 & 1.457 & .058 & -.007 & .914 \\
\hline $\begin{array}{l}\text { 5.- Participo en actividades sociales de mi barrio o } \\
\text { comunidad }\end{array}$ & 2.67 & 1.45 & -.008 & .032 & .958 \\
\hline 6.- Participo en algún grupo social & 2.54 & 1.484 & -.076 & -.020 & .849 \\
\hline $\begin{array}{l}\text { 7.- Acudo a las llamadas de apoyo de mi } \\
\text { comunidad }\end{array}$ & 3.01 & 1.455 & .121 & -.034 & .709 \\
\hline $\begin{array}{l}\text { 8.- Si tuviera problemas podría encontrar ayuda en } \\
\text { mi organización }\end{array}$ & 3.41 & 1.353 & -.110 & .837 & .094 \\
\hline $\begin{array}{l}\text { 9.- Estas organizaciones y servicios son importante } \\
\text { fuente de apoyo }\end{array}$ & 3.63 & 1.269 & .051 & .921 & -.045 \\
\hline $\begin{array}{l}\text { 10.- En caso de necesidad acudiría a estas } \\
\text { organizaciones }\end{array}$ & 3.84 & 1.240 & .080 & .743 & -.045 \\
\hline
\end{tabular}

Tabla 3. Análisis factorial confirmatorio $(\mathrm{n} 2=389)$

\begin{tabular}{|c|c|c|c|c|c|}
\hline \multirow[b]{2}{*}{ Ítems } & \multirow[t]{2}{*}{ Media } & \multirow[t]{2}{*}{ Desv. Tip. } & \multicolumn{3}{|c|}{ Saturaciones factoriales exploratorias } \\
\hline & & & Integración & Participación & Apoyo Social \\
\hline 1.- Me siento identificado con mi barrio & 3.62 & 1.20 & .837 & & \\
\hline $\begin{array}{l}\text { 2.- Mis opiniones son bien recibidas en mi } \\
\text { barrio }\end{array}$ & 3.30 & 1.26 & .816 & & \\
\hline 3.- Siento el barrio como mío & 3.53 & 1.28 & .884 & & \\
\hline $\begin{array}{l}\text { 4.- Colaboro en las organizaciones y } \\
\text { asociaciones de mi comunidad }\end{array}$ & 2.82 & 1.41 & & .904 & \\
\hline $\begin{array}{l}\text { 5.- Participo en actividades sociales de mi } \\
\text { barrio o comunidad }\end{array}$ & 2.72 & 1.39 & & .920 & \\
\hline 6.- Participo en algún grupo social & 2.67 & 1.49 & & .793 & \\
\hline $\begin{array}{l}\text { 7.- Acudo a las llamadas de apoyo de mi } \\
\text { comunidad }\end{array}$ & 3.04 & 1.42 & & .773 & \\
\hline $\begin{array}{l}\text { 8.- Si tuviera problemas podría encontrar ayuda } \\
\text { en mi organización }\end{array}$ & 3.41 & 1.34 & & & .888 \\
\hline $\begin{array}{l}\text { 9.- Estas organizaciones y servicios son } \\
\text { importante fuente de apoyo }\end{array}$ & 3.61 & 1.25 & & & .913 \\
\hline $\begin{array}{l}\text { 10.- En caso de necesidad acudiría a estas } \\
\text { organizaciones }\end{array}$ & 3.80 & 1.26 & & & .823 \\
\hline
\end{tabular}

Nota. Los valores de probabilidad de la matriz de cargas factoriales fueron estadísticamente significativos $p<.001$

factores correlacionados que en total explicaron el $82 \%$ de la varianza del constructo. Como se observa en la Tabla 2, los ítems 1, 2 y 3 coinciden con el factor denominado originalmente integración, los ítems 4, 5, 6 y 7 se agrupan dentro del factor apoyo social y los ítems 8, 9 y 10 con el factor participación. Las correlaciones entre integración y participación fueron positivas $(r=.311)$, entre integración y apoyo social positivas ( $r=.574)$ y, entre apoyo social $\mathrm{y}$ participación una correlación baja y positiva ( $r=.024)$.

\section{Análisis factorial confirmatorio}

A continuación, se sometió a prueba la estructura factorial identificada, mediante el modelamiento de ecuaciones estructurales. El modelo sometido a prueba consideró una estructura de tres factores correlacionados, los resultados revelaron un ajuste satisfactorio $\left(X^{2}\right.$ $[\mathrm{gl}=32=110.130] ; p<.001 ; \mathrm{CFI}=.989 ; \mathrm{TLI}=.984$; RMSEA=.079), ratificando los resultados de la solución factorial exploratoria y los resultados empíricos de estudios anteriores (Gracia \& Herrero, 2006). Todas las saturaciones de los ítems fueron estadísticamente significativas (ver Tabla 3), 
Tabla 4. Fiabilidad. Correlación ítem total -C.I.T- Alfa de Cronbach si se elimina el elemento -A.C.E.E-

\begin{tabular}{lcc}
\multicolumn{1}{c}{$(\mathrm{n}=777)$} & \\
\hline Items & C.I.T & A.C.E.E \\
\hline 1.- Me siento identificado con mi barrio & .755 & .773 \\
2.- Mis opiniones son bien recibidas en mi barrio & .725 & .800 \\
3.- Siento el barrio como mío & .704 & .820 \\
\hline & Alfa integración comunitaria $=.855$ \\
4.- Colaboro en las organizaciones y asociaciones de mi comunidad & .819 & .826 \\
5.- Participo en actividades sociales de mi barrio o comunidad & .827 & .823 \\
6.- Participo en algún grupo social & .683 & .879 \\
7.- Acudo a las llamadas de apoyo de mi comunidad & .677 & .880 \\
\hline & Alfa participación comunitaria $=.885$ \\
8.- Si tuviera problemas podría encontrar ayuda en mi organización & .728 & .800 \\
9.- Estas organizaciones y servicios son importante fuente de apoyo & .778 & .751 \\
10.- En caso de necesidad acudiría a estas organizaciones & .682 & .839
\end{tabular}

además se confirmó una correlación positiva entre la dimensión integración y participación ( $r=.617)$, una correlación positiva entre integración y apoyo social $(r=.414)$ y correlación positiva entre participación y apoyo social $(r=.262)$.

\section{Análisis de fiabilidad}

Finalmente se evaluó la fiabilidad de los factores, mediante los coeficientes Alfa de Cronbach y el coeficiente Omega de McDonald, dispuesto entre paréntesis. Para los tres factores se observan coeficientes de fiabilidad aceptables; Integración comunitaria alfa $=.855$ (.933), Participación comunitaria alfa $=.885(.950) \mathrm{y}$ Apoyo social alfa $=.855$ (.948). Además, se presentan los índices de homogeneidad medidos a través del procedimiento de correlación ítem total (ver Tabla 4), esta medida osciló entre .677 y .827, mostrando el aporte de cada ítem a la medición de un rasgo común. El procedimiento de alfa si se elimina el elemento, arrojó resultados favorables, que indican que cada uno de los ítems aporta significativamente a la fiabilidad del instrumento.

\section{Discusión}

Este artículo tuvo como objetivo evaluar las propiedades psicométricas del cuestionario de apoyo comunitario en una muestra de personas mayores chilenas, analizando su validez de constructo, fiabilidad por consistencia interna y homogeneidad.
Los resultados de este estudio confirman la estructura de tres factores correlacionados, evidencia que se suma a los hallazgos anteriores (Herrero \& Gracia, 2007; Gracia \& Herrero, 2006). Los factores denominados integración comunitaria, apoyo social e integración comunitaria explicaron en conjunto el $82 \%$ de la varianza del constructo. Cabe señalar que la varianza total explicada en esta investigación es mayor a la obtenida originalmente por los autores del cuestionario, que era de $48.88 \%$ (Gracia \& Herrero, 2006). La estabilidad de esta solución fue ratificada a través del modelamiento de ecuaciones estructurales, los índices de bondad de ajuste ratificaron que el modelo de tres factores correlacionados ajustó adecuadamente a los datos, con indicadores robustos de ajuste absoluto y relativo. Finalmente, el análisis de fiabilidad por consistencia interna, tanto para datos continuos como para datos ordinales, arrojó valores adecuados para cada uno de los indicadores.

Esta investigación presenta algunas limitaciones relacionadas con las características de la muestra. En este estudio participaron personas mayores jóvenes (la mayoría tiene menos de 70 años) con una baja prevalencia de enfermedades funcionales que les permiten mantener activos y vinculados fuertemente con redes comunitarias, por lo que se debiera analizar el impacto del apoyo social de la comunidad en personas mayores con una edad más avanzada y que posiblemente se vinculen menos con la comunidad de vecinos, pero posiblemente más 
con las redes formales de apoyo (centro de salud y otros servicios públicos de atención geriátrica/gerontológica). Igualmente, el hecho de que se trata de una muestra joven y activamente participativa en su entorno comunitario, puede influir en que sus respuestas fueran sesgadas o bien orientadas a la deseabilidad social, es decir, los entrevistados mayores tienden a presentarse a sí mismos de forma favorable ante los profesionales de las ciencias sociales (Vigil-Colet, Morales-Vives, \& Lorenzo-Seva, 2013). Como futuras líneas de investigación se plantea aplicar este cuestionario en otros grupos de edad y otros colectivos en Chile, como es el caso de los jóvenes (entre 25-35 años) que se integran en diversas iniciativas comunitarias tras la obtención de una vivienda o tras la emancipación de sus hogares con la intención de integrase al barrio/vecindario. Igualmente podría ser interesante aplicar este instrumento en los colectivos de migrantes (colombianos, haitianos y peruanos) con objeto de evaluar su integración social/comunitaria.

Retomando los aspectos de aplicabilidad del cuestionario de apoyo social comunitario (Gracia, Herrero, \& Musitu, 2002), se trata de una herramienta de intervención gerontológica que puede ser utilizada, tanto en el diagnóstico como en la intervención en sí misma, para la valoración de la integración social de las personas mayores. El aislamiento social o la falta de redes sociales repercute negativamente en la salud física y mental (Rivas-Diez, 2013), es decir, es un factor de riesgo que debe ser evaluado en edades avanzadas.

Desde el modelo de intervención centrado en las redes sociales de apoyo y específicamente las redes comunitarias, tiene como objetivo "la creación de un proceso de interacción con el entorno social que se capaz de satisfacer las necesidades psicosociales de las personas" (Gracia, 1997:39). A lo que se añade, que el nivel de análisis del apoyo social comunitario es distinto al que ofrecen las relaciones íntimas o de confianza, se trata más bien un espacio de relaciones más amplio y más diverso (Gracia y Herrero, 2006). Por tanto, la valoración de las redes comunitarias en la vejez implica analizar la interacción o integración con el entorno social (barrio, vecindario, organizaciones sociales, etc.) y el acceso a un sistema de apoyo más amplio, diverso y que eventualmente, pueden dar una respuesta más específica a las necesidades emocionales, instrumentales e informativas de las personas mayores.

\section{Referencias}

Antonucci, T. C., Ajrouch, K. J., \& Birditt, K. S. (2014). The convoy model: Explaining social relations from a multidisciplinary perspective. The Gerontologist, 54(1), 82-92. doi:10.1093/geront/gnt1 18

Arechabala, M. C., \& Miranda, C. (2002). Validación de una escala de apoyo social percibido en un grupo de adultos mayores adscritos a un programa de hipertensión de la región metropolitana. Ciencia y Enfermería, 8(1), 49-55. doi:10.4067/S071795532002000100007

Brown, S. C., Mason, C. A., Spokane, A. R., Cruza-Guet, M. C., Lopez, B., \& Szapocznik, J. (2009). The relationship of neighborhood climate to perceived social support and mental health in older Hispanic immigrants in Miami, Florida. Journal of Aging and Health, 21(3), 431-459. doi:10.1177/0898264308328976

Browne, M., \& Cudeck, R. (1993). Alternative ways of assessing model fit. In: K., Bollen \& J., Long (Eds.) Testing Structural Equation Models (pp. 136-162). Beverly Hills, CA: Sage.

Bunout, D., Osorio, P., Barrera, G., Torrejón, M. J., Meersohn, C., Anigstein, M. S., ... \& de la Maza, M. P. (2012). Quality of life of older Chilean subjects living in metropolitan Santiago, Chile. Influence of socioeconomic status. Ageing Research, 3(1), 3.

Burholt, V., \& Dobbs, C. (2014). A support network typology for application in older populations with a preponderance of multigenerational households. Ageing and society, 34(07), 1142-1169. doi:10.1017/S0144686X12001511

Campos, F., Herrera, S., Fernández, B., \& Valenzuela, E. (2014). Chile y sus mayores. Resultados tercera encuesta nacional Calidad de Vida en la Vejez 2013. Santiago: Servicio Nacional del Adulto Mayor, Pontificia 
Universidad Católica de Chile y Caja de Compensación Los Andes.

Carstensen, L. L., Isaacowitz, D. M., \& Charles, S. T. (1999). Taking time seriously. A theory of socioemotional selectivity. American Psychologist, 54, 165-181.

Castillo-Carniglia, A., Albala, C., Gangour, A. D., \& Uauy, R. (2012). Factores asociados a satisfacción vital en una cohorte de adultos mayores de Santiago, Chile. Gaceta Sanitaria, 26(5), 414-20. doi:10.1016/j.gaceta.2011.11.021

Chappell, N. L., \& Funk, L. M. (2011). Social support, caregiving and aging. Canadian Journal on Aging, 30, 355-70. doi:10.1017/S0714980811000316

Cheng, S. T., Lee, C. K., Chan, A. C., Leung, E. M., \& Lee, J. J. (2009). Social network types and subjective well-being in Chinese older adults. The Journals of Gerontology Series B: Psychological Sciences and Social Sciences, 64B (6), 713-722. doi:10.1093/geronb/gbp075

Cloutier-Fisher, D., Kobayashi, K., \& Smith, A. (2011). The subjective dimension of social isolation: A qualitative investigation of older adults' experiences in small social support networks. Journal of Aging Studies, 25(4), 407-414. doi:10.1016/j.jaging.2011.03.012

Domínguez-Guedea, M. T. (2016). Bienestar en cuidadores familiares de adultos mayores: Un Derecho, una Aspiración y un Constructo Psicológico. Revista Iberoamericana de Diagnóstico y Evaluación - e Avaliação Psicológica, 41(1).

Fiori, K. L., Antonucci, T. C., \& Akiyama, H. (2008). Profiles of social relations among older adults: A cross-cultural approach. Ageing and Society, 28(02), 203231. doi:10.1017/S0144686X07006472

Fiori, K. L., Smith, J., \& Antonucci, T. C. (2007). Social network types among older adults: A multidimensional approach. The Journals of Gerontology Series B: Psychological Sciences and Social Sciences, 62(6), P322-P330.

Flora, D., \& Curran, P. (2004). An empirical evaluation of alternative methods of estimation for confirmatory factor analysis with ordinal data. Psychological Methods, 9(4), 466-491. doi:10.1037/1082-989X.9.4.466

Freiberg, A., Stover, J., de la Iglesia, G., \& Fernández, M. (2013). Correlaciones policóricas y tetracóricas en estudios factoriales exploratorias y confirmatorias. Revista de Ciencias Psicológicas, 7(2), 151164.

Gallardo-Peralta, L. P. (2013). Redes de apoyo social en personas mayores chilenas. Aspectos estructurales y funcionales. Revista Trabajo Social, 89, 9-18.

Gallardo-Peralta, L. (2017). The relationship between religiosity/spirituality, social support, and quality of life among elderly Chilean people. International Social Work, 60 (6), 1498-1511. doi:10.1177/0020872817702433

Gracia, E. (1997). El apoyo social en la intervención comunitaria. Barcelona: Paidós.

Gracia, E., \& Herrero, J. (2006). La comunidad como fuente de apoyo social: Evaluación e implicaciones en los ámbitos individual y comunitario. Revista Latinoamericana de Psicología, 38(2), 327-342.

Gracia, E., Herrero, J., \& Musitu, G. (2002). Evaluación de recursos y estresores psicosociales en la comunidad. Madrid: Síntesis.

Hand, C., Law, M., Hanna, S., Elliott, S., \& McColl, M. A. (2012). Neighbourhood influences on participation in activities among older adults with chronic health conditions. Health y Place, 18, 869-876. doi:10.1016/j.healthplace.2012.03.004

Heinze, J. E., Kruger, D. J., Reischl, T. M., Cupal, S., \& Zimmerman, M. A. (2015). Relationships among disease, social support, and perceived health: A lifespan approach. American Journal of Community Psychology, 56(3-4), 268-279. doi:10.1007/s10464-015-9758-3

Herrera, M. S., Elgueta, R. P., \& Fernández, M.B. (2014). Capital social, participación en asociaciones y satisfacción personal de las personas mayores en Chile. Revista de Saúde Pública, 48(5), 739-749. doi:10.1590/S0034-8910.2014048004759

Herrera, M. S., Barros, C. \& Fernández, M. B. (2011). Predictors of quality of life in old age: A multivariate study in Chile. Journal of Population Ageing, 4, 121-139. doi:10.1007/s12062-011-9043-7

Herrero, J., \& Gracia, E. (2007). Measuring perceived community support: Factorial 
structure, longitudinal invariance, and predictive validity of the PCSQ (Perceived Community Support Questionnaire). Journal of Community Psychology, 35(2), 197-217.

Lang, F. R., \& Carstensen, L. L. (1994). Close emotional relationships in late life: Further support for proactive aging in the social domain. Psychology and Aging, 9, 315-324.

Li, T., \& Zhang, Y. (2015). Social network types and the health of older adults: Exploring reciprocal associations. Social Science \& Medicine, 130, 59-68. doi:10.1016/j.socscimed.2015.02.007

Litwin, H., \& Shiovitz-Ezra, S. (2011). Social network type and subjective well-being in a national sample of older Americans. The Gerontologist, 51(3), 379-388. doi:10.1093/geront/gnq094

Lorenzo-Seva, U. (1999). Promin: A method for oblique factor rotation. Multivariate Behavioral Research, 34(3), 347-356. doi:10.1207/S15327906MBR3403_3

Lorenzo-Seva, U., \& Ferrando, P. J. (2006). FACTOR: A computer program to fit the exploratory factor analysis model. Behavior research methods, 38(1), 88-91.

Lorenzo-Seva, U., Timmerman, M. E., \& Kiers, H.A.L. (2011). The Hull method for selecting the number of common factors. Multivariate Behavioral Research, 46, 340-364.

McDonald, R. (1999). Test Theory: A unified treatment. Mahwah, NJ: Lawrence Erlbaum Associates.

Mella, R., González, L., D'appolonio, J., Maldonado, I., Fuenzalida, A., \& Díaz, A. (2004). Factores asociados al bienestar subjetivo en el adulto mayor. Psykhe, 13, 7989. doi:10.4067/S0718-22282004000100007

Ministerio de Desarrollo Social (2015). Encuesta Nacional de Caracterización socioeconómica, CASEN. Santiago de Chile: Ministerio de Desarrollo Social

Muthén, L. \& Muthén, B. (1998-2012). Mplus User's Guide (7.a ed.). Los Angeles, CA: Muthén \& Muthén.

Nguyen, A. W. (2017). Variations in social network type membership among older African Americans, Caribbean Blacks, and Non-Hispanic Whites. The Journals of
Gerontology. Series B, Psychological Sciences and Social Sciences. doi:10.1093/geronb/gbx016

Ortiz, M., \& Baeza, M. J. (2011). Psychometric properties of a scale designed to measure perceived social support in chilean patients with Type 2 Diabetes. Universitas Psychologica, 10(1), 189-196.

Rivas-Diez, R. (2013). Apoyo social funcional en mujeres de la población general y en mujeres maltratadas chilenas. Propiedades psicométricas del Duke-UNC-11. Revista Iberoamericana de Diagnóstico y Evaluación - Avaliação Psicológica, 2(36).

Schumacher, R., \& Lomax, R. (1996). A beginner's guide to structural equation modeling. New Jersey: Lawrence Erlbaum Associates, Publishers.

Thoits, P. A. (2011). Mechanisms linking social ties and support to physical and mental health. Journal of Health and Social Behavior, 52, 145-161. doi:10.1177/0022146510395592

Torres, M., Quezada, M., Rioseco, R., \& Ducci, M.E. (2008). Calidad de vida de adultos mayores pobres de viviendas básicas: Estudio comparativo mediante uso de WHOQolBREF. Revista Médica de Chile, 136, 325333. doi:10.4067/S0034-98872008000300007

Trizano-Hermosilla, I., \& Alvarado, J. M. (2016). Best alternatives to Cronbach's Alpha reliability in realistic conditions: Congeneric and asymmetrical measurements. Frontiers in Psychology, 7, 1-8. doi:10.3389/fpsyg.2016.00769 Urzúa, A., Bravo, M., Ogalde, M. \& Vargas, C. (2011). Factores vinculados a la calidad de vida en la adultez mayor. Revista Médica de Chile, 139(8), 1006-1014. doi:10.4067/S0034-98872011000800005

van Bilsen, P. M. A., Hamers, J. P. H., Groot, W., \& Spreeuwenberg, C. (2008). The use of community-based social services by elderly people at risk of institutionalization: An evaluation. Health Policy, 87(3), 285-295. doi:10.1016/j.healthpol.2007.12.015

Vigil-Colet, A., Morales-Vives, F., \& LorenzoSeva; U. (2013). How social desirability and acquiescence affect the age-personality relationship. Psicothema, 25(3), 342-348. doi:10.7334/psicothema2012.297

Vivaldi, F., \& Barra, E. (2012). Bienestar psicológico, apoyo social percibido y 
percepción de salud en adultos mayores. Terapia Psicológica, 30(2), 23-29. doi:10.4067/S0718-48082012000200002

Zimet, G. D., Dahlem, N. W., Zimet, S. G., \& Farley, S. G. (1988). The Multidimensional Scale of Perceived Social Support. Journal of Personality Assessment, 52(1), 30-41.

doi:10.1207/s15327752jpa5201_2 